\title{
An Administrative system of a Software Platform for Cloud Computing and Development of Intelligent Services
}

\author{
Philip Moskalenko, Elena Shalfeyeva, Vadim Timchenko \\ Lab of intelligent systems \\ Institute for Automation and Control Processes FEB RAS \\ Vladivostok, Russian Federation \\ philipmm@iacp.dvo.ru
}

\begin{abstract}
A concept of a system for support of administrative control is presented. The system supports accumulation and cooperative development of information and software components of intelligent services which are developed and used in IACPaaS software platform.
\end{abstract}

Keywords-cloud computing; administrative system; user control; software management

\section{INTRODUCTION}

Cloud computing technology is widely used and evolving fast today $[1,2]$. Its main idea is to provide tools for implementation and usage of software as internetservices. Environments of modern cloud computing platforms do not provide development and support of intelligent software systems which usually consist of task solver, user interface and a knowledge base. This usually meets these difficulties: a) specialists of various types (domain experts, knowledge engineers, software developers etc.) are involved into it; b) creation, development and support of such components as knowledge bases and other complex-structured data are too labour-intensive.

The mechanisms for centralized (cloud) control of all components of intelligent internet-services [3, 4] are intended to provide a long-term usage of such services and cooperative activities of their users, domain experts, managers and developers. In order to achieve this goal a concept of accumulation and evolution of both - intelligent services and their separate components which can be used for creation of new applied and system services - is proposed. These components are: information resources of different levels of abstraction (knowledge bases, databases, ontologies, meta-ontologies) and software units (agents [5], template components of interface).

This paper describes a concept of administrative system which is one of the mechanisms for:

- providing a controlled access to functionality of software platform for cloud computing «Intelligent Applications, Control and Platform as a Service» (IACPaaS) [4];

- control of users' privileges for access and usage of apllied and system services which were developed with the use of this platform;
- control for accumulation and collective development of both - information resources and software components of intelligent services.

\section{Circulation OF REQUests}

Administrative tools which provide management for data warehouse contents, users and their privileges, control of collective development of components are based on circulation of requests between users and administrators of the software platform. The implementation of requests' circulation is based on processing of "inner" information resources of administrative system: "Data warehouse structure", "Users and authorities", "Requests".

The following types of requests are present:

- registration of a user in a domain (either in an existing or in a new one);

- data warehouse modification (creation of a storage unit, creation of a version of a storage unit, change of a state of a version of a storage unit);

- acquiring of an authority to use an internet-service with certain information resources.

The circulation of request goes as the following:

1) request creation;

2) request queuing:

a) a request for registration in an existing domain goes into the queue of requests for its administrator;

b) a request for registration in a new domain goes into the queue of requests for the platform administrator;

c) a request for acquiring of an authority in some domain goes into the queue of requests for its administrator;

d) a request for data warehouse modification in some domain goes through 2-step queuing: first it goes into the queue of requests for the administrator of this domain, then (if case of approval) it goes into the queue of requests for the administrator of the IACPaaS software platform;

3) request examination: when an administrator examines a request he can either approve it (then a user gets registration in a domain, or he gets an authority, or the data warehouse becomes modified), or reject, or return for rework. 


\section{INNER INFORMATION RESOURCES}

\section{A. Data warehouse contents}

Information resource "Data warehouse contents" contains:

- a set of domains;

- a link to a user who is the manager of the IACPaaS software platform;

- a link to a user who is the administrator of the IACPaaS software platform;

- a set of links to requests which are in queue for IACPaaS administrator.

Each domain is presented by its name, description, contents, link to a user who is its administrator and a set of links to requests which are in queue for him. The contents of the domain are a set of sections. Each section is presented by its name and description and contains storage units of various types (information resources, services, agents etc.).

\section{B. Users and authorities}

Information resource "Users and authorities" stores the information about users. Each user is described with:

- general information (id, login, name, e-mail, etc.)

- a flag that indicates whether he is an IACPaaS manager or not;

- a flag that indicates whether he is an IACPaaS administrator or not;

- a set of links to requests which were created by this user;

- a set of his authorities in domains where he is registered.

Each element of that set contains:

- a link to the domain (see "Data warehouse contents");

- a flag that indicates whether he is an administratorof this domain or not;

- $\quad$ a set authorities which he can use.

\section{Requests}

Information resource "Requests" stores general information and contents of requests.

General information consists of the following:

- date of request creation;

- basis of request;

- status of request;

- administrator's comment for rework (filled in by some administrator when the request is being returned for rework)

Contents of a request vary by its type.

\section{IMPLEMENTATION}

Functionality of each administrative tool is implemented in agent "Controller", which receives the messages from the Web-agent which is a part of IACPaaS software platform and which interacts with the Web-server. "Controller" performs necessary read/write operations in the "inner" information resources. If the results of its work should be shown to a user - it forms an abstract user interface description with the use of platform API methods and sends it to Web-agent in a message.

\section{CONCLUSION}

In contrast to other administrative systems, which are used in software-and-information systems and which implement some policy for control of users and their privileges and of access to resources, the developed one is designed to support a concept of accumulation and development of applied and system services and their separate components. The latter are information resources of different levels of abstraction, agents (which are parts of task solvers and which can perform domain-independent calculations), operations on information resources (which preserve their integrity), template components of user interface (which can be domain-dependent or domainindependent). They all can be used for development of new applied and system intelligent services. As any usage of a service is controlled by a domain administrator so the system also introduces a mechanism of control for the usage of development tools.

\section{ACKNOWLEDGMENT}

The work was performed with a financial support of RFBR (project 13-07-00024) and FEB RAS (project 12-IIUB-01I-001).

\section{REFERENCES}

[1] T. Koncer, "Cloud computing: all as a service," PC Week/RE, №32 (638), 2008, Electronic Publication: http://www.pcweek.ru/themes/ detail.php?ID=112879/ (in Russian).

[2] M. Kalkul, "Clear sky till the horizon: cloud computing and security from the cloud," Electronic Publication: http://www.securelist.com/ ru/analysis/204007652/Yasnoe nebo do samogo gorizonta oblachn ye_vychisleniya_i_bezopasnost_iz_oblaka/ (in Russian).

[3] V. Gribova et al., "A cloud platform for development and control of intelligent systems," Proc. of International scienec-technoical conference OSTIS-2011, Minsk, BSUIR, pp. 5-14. (in Russian).

[4] V. Gribova et al., "IACPaaS project. A software platform for intelligent systems based on cloud computing," Artificial intelligence and decision-making, №1, 2011, pp. 27-35. (in Russian).

[5] V. Gribova et al., "Agent-oriented approach for the development of intelligent internet-services," // Proc. of Congress on intelligent systems and information technologies (IS\&IT'12), Divnomorskoe, Russian Federation, 2012. vol.1, pp.218-223. (in Russian). 